Sensors 2002, 2, 41-49

\title{
sensors
}

ISSN 1424-8220

(C) 2002 by MDPI

http://www.mdpi.net/sensors

\section{Studies on the Electrochemical Behaviour of Hydroquinone at L-cysteine Self-Assembled Monolayers Modified Gold Electrode}

\author{
Shengfu Wang* and Dan Du \\ College of Chemistry and Material Science, Hubei University, 430062, Wuhan, People's Republic of \\ China. Tel: 027-88664302. \\ * Author to whom correspondence should be addressed. E -mail: danlinda@,263.net
}

Received: 7 January 2002 / Accepted: 21 January 2002 / Published: 4 February 2002

\begin{abstract}
L-Cysteine is combined onto gold electrode to form a self-assembled monolayers modified electrode (L-Cys/Au SAMs) by taking advantage of strong sulfur-gold interaction. ATR-FTIR, SEM, cyclic voltammetry (CV) and impedance were used for the characterization of the film. It shows excellent stability upon voltametric scanning and a good voltametric response towards hydroquinone with the potential ranged from 0.8 to -0.2 $\mathrm{V}$ (vs.SCE) in $0.5 \mathrm{M}$ HAc-NaAc buffer solution ( $\mathrm{pH} 4.8$ ). The oxidation potential of hydroquinone on the modified electrode shifted negatively about $330 \mathrm{mV}$ as compared with the bare gold electrode. The plot of catalytic current vs.its concentration has a good linear relation in the range of $2.0 \times 10^{-6} \sim 2.0 \times 10^{-4} \mathrm{M}$ with the correlation coefficient of 0.9986 and the detection limit of $4.0 \times 10^{-7} \mathrm{M}$ by different pulse voltammetry (DPV). Mechanism for the electrocatalytical process has been studied.
\end{abstract}

Keywords: Self-assembled monolayers, ATR-FTIR, SEM, L-cysteine, Hydroquinone

\section{Introduction}

Self-assembled monolayers (SAMs) are ordered molecular assemblies formed by the adsorption of an active surfactant on a solid surface ${ }^{[1]}$. This simple process makes SAMs inherently manufacturable and thus technologically attractive for building super lattices and for surface engineering. The use of SAMs in various fields is rapidly growing; especially organic thiols chemisorbed on gold are particularly suitable for electrochemical and chemical studies due to their excellent stability and highly characterized structures ${ }^{[2 \sim 6]}$. For example, Rubinstein and coworkers ${ }^{[7 \sim 9]}$ demonstrated that mixed functionalized SAMs could recognize copper ions selectively in 
the presence of other ions. Mandler and co-workers ${ }^{[10,11]}$ have reported a highly sensitive electrode for cadmium ions and chromium(VI) ions using self-assembled monolayers-based electrodes. S.Berchmans et al ${ }^{[12]}$ studied the formation of nickel hydroxide monolayers on Au through a SAMs, J.Y.Mo ${ }^{[13]}$ presented a method for the determination of dopamine and epinephrine by SAMs. At the same time, SAMs have been used for preparation of electrochemical biosensors. Willner ${ }^{[14]}$ Creager $^{[15]}$ and Wilson ${ }^{[16]}$ reported on the design of sensors for glucose based on incorporating glucose oxidase into SAMs. In this paper, We employed L-cysteine $\left(\mathrm{HSCH}_{2} \mathrm{CH}\left(\mathrm{NH}_{2}\right) \mathrm{COOH}\right)$ to modify the gold surface for the determination of hydroquinone in photographic imagine effluents because XPS ${ }^{[17]}$ and FT-IR ${ }^{[18]}$ spectroscopic studies had shown that cysteine could bind onto the gold surface through the strong sulfer-gold interaction and the surface was thus functionalized with amino acid ${ }^{[19]}$. Because sulfur-gold interactions are strong, the monolayers can be assembled in a short time and are reasonably stable upon cyclic voltammetric scanning in buffer solutions.

Documents have shown that L-cysteine can accelerate the electron transfer rate of sub-ppb copper(II) ${ }^{[20]}$ hemoglobin ${ }^{[21]}$ dopamine $^{[22]}$ and superoxide dismutase ${ }^{[23]}$. We observed that L-cysteine SAMs also has striking electrocatalysis to hydroquinone. Due to the wide existence in photographic imaging agent, monomer inhibitor, coating, stabilizer, antioxidant, auxiliaries and so on, hydroquinone has normally been determined by spectrophotography, which is troublesome by drawing curved line. In our experiment, hydroquinone has a good response at L-Cys/Au SAMs. The oxidation potential shifted negatively by about $330 \mathrm{mV}$ compared with bare gold electrode. The catalytic current vs. its concentration has a good linear relation in the range of $2.0 \times 10^{-6} \sim 2.0 \times 10^{-4} \mathrm{M}$ with the correlation coefficient of 0.9986 and the detection limit of $4.0 \times 10^{-7} \mathrm{M}$ by DPV. The proposed procedure has been applied to the determination of hydroquinone in some practical samples, such as photographic imagine effluents, with a simple-rapid and accurate result.

\section{Experimental}

\section{Apparatus}

Electrochemical measurements were preformed with a three-electrode system comprising a bare gold electrode or L-cysteine self-assembled monolayers modified gold electrode (i.e. L-Cys/Au SAMs) as the working electrode, a saturated calomel electrode (SCE) as the reference electrode and a platinum sheet as the counter electrode. The electrodes were connected to CHI630A (U.S.A). All potentials given in this paper are referred to the SCE. FTIR spectrometer instrument was purchased from Co. Perking Elmen. All the spectra were obtained with an average of 100 scan with $4 \mathrm{~cm}^{-1}$ resolutions. SEM photographs were taken on the instrument of HITACHI S-570. The experimental results are obtained at room temperature.

\section{Reagents}

L-cysteine (biochemical reagent, Shanghai, China, not necessary be purified before using), polishing powder $(0.05 \mu \mathrm{m})$, other chemicals are of analytical grade or better. Experimental water used is doubly distilled water. 


\section{Preparation of L-Cys/Au SAMs}

The bare gold electrode was polished to a mirror-like surface with $0.05 \mu \mathrm{m} \alpha-\mathrm{Al}_{2} \mathrm{O}_{3}$ and immersed in Piranha solution (a hot mixed solution of $30 \% \mathrm{H}_{2} \mathrm{O}_{2}$ and concentrated $\mathrm{H}_{2} \mathrm{SO}_{4}$, volume ratio 1:3), then rinsed ultrasonically with water and absolute ethanol and sonicated in twice-distilled water. This electrode was voltammetrically cycled and characterized in $0.5 \mathrm{M} \mathrm{H}_{2} \mathrm{SO}_{4}$ until a stable cyclic voltammogram was obtained. L-Cys/Au SAMs was prepared by soaking the clean gold electrode in $0.05 \mathrm{ML}$-cysteine solution for $24 \mathrm{~h}$.

\section{Results and Discussion}

FTIR of L-Cys/Au SAMs

Fig.1 shows the FTIR spectra of crystalline L-cysteine (1a) and L-cysteine film modified gold electrode (1b). Comparing with the two spectra, the most important difference between them is that the band at $2550 \mathrm{~cm}^{-1}$ of $-\mathrm{SH}$ in Fig.1a disappeared in Fig. 1b. It is attributed to the cleavage of S-H bond and the formation of a new bond, i.e., S-Au bond. This phenomenon proved the fact that L-cysteine has been assembled on the gold electrode. Both Fig.1a and Fig.1b showed a set of peaks between $2600 \sim 3160 \mathrm{~cm}^{-1}$ corresponding to the stretching mode of ammonium. The band corresponding to the $\mathrm{COO}^{-}$asymmetric stretching mode expected to be at about $1600 \mathrm{~cm}^{-1}$ is not clearly seen but could be obscured by the $\mathrm{NH}_{2} / \mathrm{NH}_{3}{ }^{+}$symmetric deformation mode at $1580 \mathrm{~cm}^{-1}{ }^{[24]}$. As the bond of $\mathrm{S}-\mathrm{Au}$ is strong, other groups in the L-cysteine molecule do not compete with it.

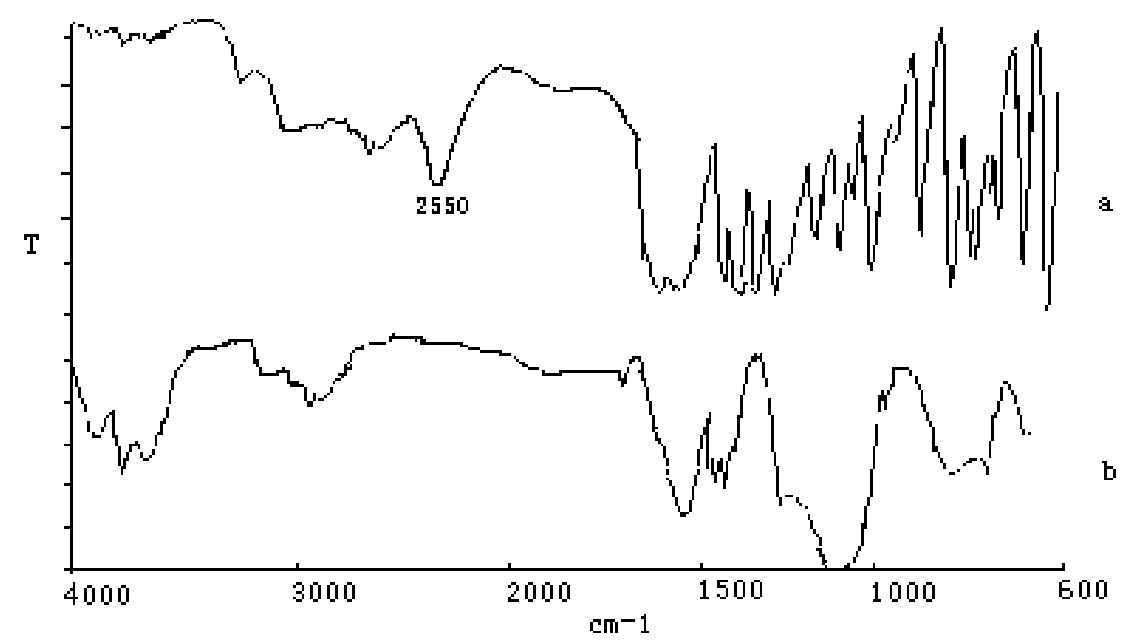

Figure 1. FTIR of L-Cysteine(a) and L-Cys/Au SAMs(b).

SEM of L-Cys/Au SAMs

The presence of the L-Cys/Au SAMs film on a gold electrode surface can be further confirmed by SEM photograph (Fig.2). So many defects were observed on bare gold electrode surface (2a) while a layer of film was obtained on L-Cys/Au SAMs (2b). Although there were still a small amount of 
drawbacks on SAMs film, a clear layer of film was obvious. It indicated that L-cysteine molecule has been assembled on the gold surface, corresponding to the result from FTIR.
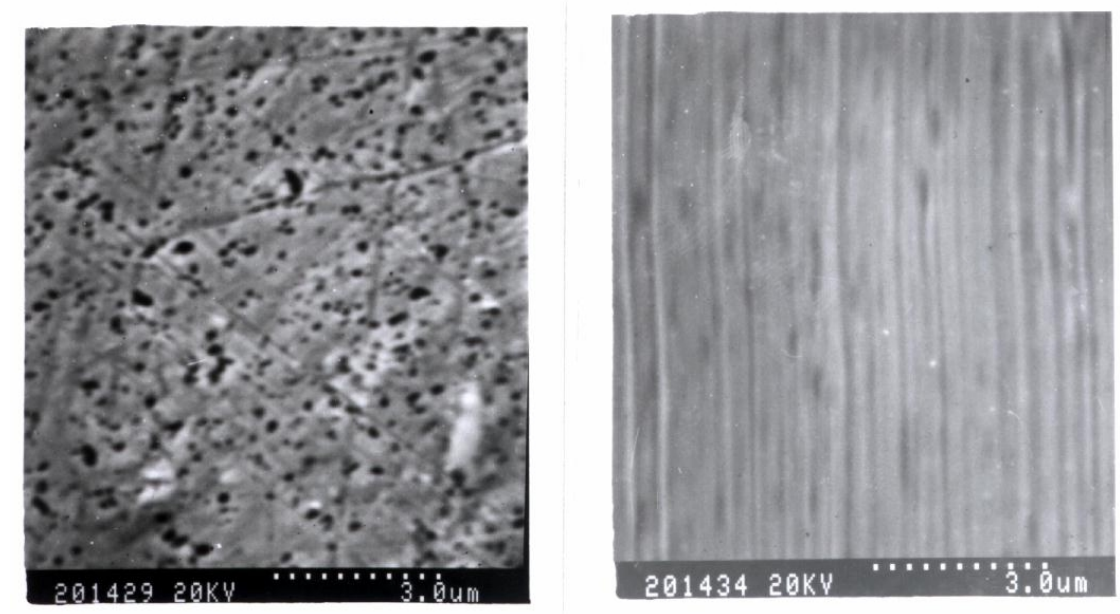

Figure 2. SEM photographs of bare gold electrode (a) and L-Cys/Au SAMs electrode (b).

\section{Electrochemical behavior of L-Cys/Au SAMs}

No redox peaks on cyclic voltammograms when L-Cys/Au SAMs is scanning in 0.5 M HAc-NaAc buffer solution ( $\mathrm{pH} 4.8$ ) with the potential ranged from 0.8 to $-0.2 \mathrm{~V}$. Comparing to that at bare gold electrode, the background current of L-Cys/Au SAMs decreases greatly. This is because L-cysteine formed monolayers at gold surface; the transfer rate between electrode surface and buffer solution was much slower ${ }^{[21]}$.

A majority of papers have confirmed that there is a strong affinity between sulfur atom and gold surface, sulfur and selenium compounds can form monolayers on gold ${ }^{[14,15]}$. Because of the existence of $-\mathrm{SH}$ in L-cysteine molecule $\left(\mathrm{SHCH}_{2} \mathrm{CH}\left(\mathrm{NH}_{2}\right) \mathrm{COOH}\right)$, this molecule can be adsorbed on gold electrode naturally, forming self-assembled monolayers.

\section{Impedance analysis in the presence of $\mathrm{Fe}(\mathrm{CN})_{6}^{3-/ 4-}$}

The ac impedance method is based upon a measurement of the response of the electrochemical cell to a small-amplitude alternating potential. The response is often shown in the complex-impedance presentation, and the result can be interpreted in terms of an equivalent electrical circuit. The surface changes of the electrode must cause a change in the ac response; this change can be understood according to Randles' equivalent circuit ${ }^{[25]}$, and can be used to estimate the electrode coverage and some kinetic parameter, such as the charge-transfer rate constant, and the dielectric constant of the monolayer film.

In terms of the Randles' equivalent circuit, two frequency regions, as at high and low, can be distinguished to understand the change in faradaic impedance. Our attention is focused on the more interesting part of the spectrum at a high frequency where the electrode reaction is purely kinetically controlled, and the heterogeneous charge-transfer resistance is expected to increase due to inhibition of 
the electron transfer by the monolayer on the electrode surface ${ }^{[26]}$. The electrode coverage $(\theta)$ is a key factor which can be used to estimate the surface state of the electrode, and the charge-transfer resistance is also related to it. Assuming that all the current is passed by pinholes on the electrode, the electrode coverage can be calculated by ${ }^{[27]}:(1-\theta)=\mathrm{R}_{\mathrm{ct}}{ }^{0} / \mathrm{R}_{\mathrm{ct}}$, where $\mathrm{R}_{\mathrm{ct}}{ }^{0}$ is the charge-transfer resistance at bare gold and $R_{c t}$ is the charge-transfer resistance at the monolayer-covered electrode under the same condition.

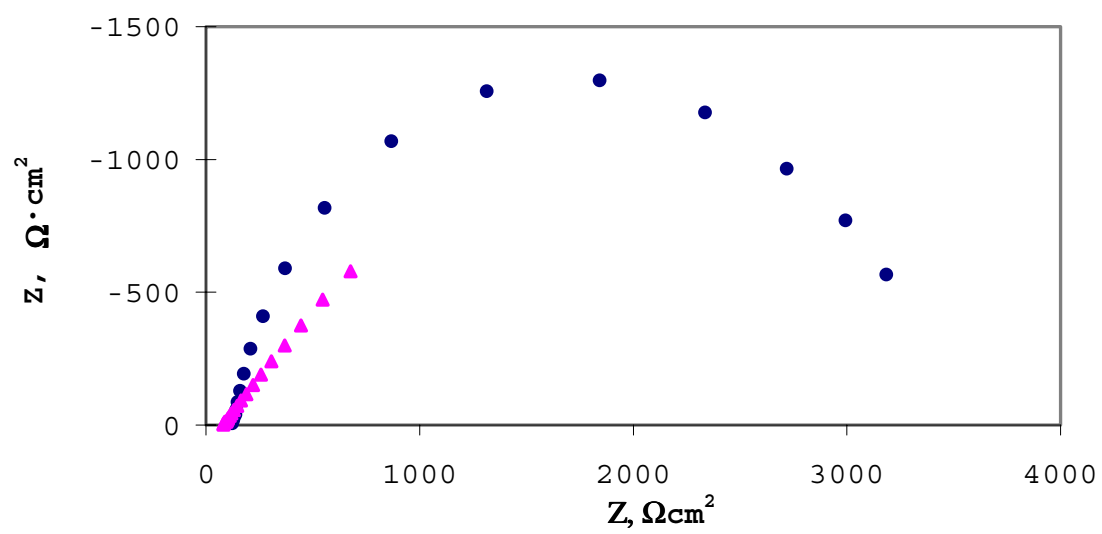

Figure 3. Complex-impedance plots measured in $1.0 \mathrm{mM} \mathrm{K}_{4}\left[\mathrm{Fe}(\mathrm{CN})_{6}\right]+1.0 \mathrm{mM} \mathrm{K}_{3}\left[\mathrm{Fe}(\mathrm{CN})_{6}\right]$ $+0.5 \mathrm{MKNO}_{3}$ at a bias potential of $0.22 \mathrm{~V}$ (vs.SCE) for bare gold electrode (a) and L-Cys modified electrode (b).

Fig. 3 is complex-impedance plot of L-Cys/Au SAMs. A comparison of complex impedance plots of a bare electrode and a monolayer-covered gold electrode shows the effect of the absorbed L-Cys monolayers on the ac response. For a monolayer-covered electrode, $\mathrm{R}_{\mathrm{ct}}$, which is the diameter of the semicirele at high frequency, is clearly greater than $\mathrm{R}_{\mathrm{ct}}$ due to an inhibition of L-Cys/Au SAMs to electron transfer. It is worth noting that the semicircle measured at bare gold is poorly defined, due to a fast electrode reaction, while it is well defined at the monolayer-coated electrode. From an analysis of the spectrum shown in Fig.3, the charge-transfer resistance is $3232 \Omega \mathrm{cm}^{2}$, the corresponding electrode coverage value was estimated to be $99.4 \%$. The charge-transfer resistance for bare gold was measured to be $20.4 \Omega \mathrm{cm}^{2}$.

\section{Electrochemical properties of hydroquinone on L-Cys/Au SAMs}

A pair of redox peaks of hydroquinone on L-Cys/Au SAMs (Fig.4a) was observed comparing with small peaks on bare gold electrode (Fig.4b) at the same concentration of hydroquinone. The oxidation potential shifted negatively by about $330 \mathrm{mV}$ from $0.657 \mathrm{~V}$ to $0.329 \mathrm{~V}$, and the peak current enhanced several times because L-cysteine accelerated electron transfer rate between hydroquinone and electrode. The peak current increased linearly with the square root of scan rate, as expected for a diffusion-controlled process, with the regression equation of $\mathrm{i}_{\mathrm{p}}=0.556 v^{1 / 2}+1.04\left(\mathrm{i}_{\mathrm{p}}: \mu \mathrm{A}, v: \mathrm{mVs}^{-1}\right.$, $\mathrm{r}=0.9972$ ). 


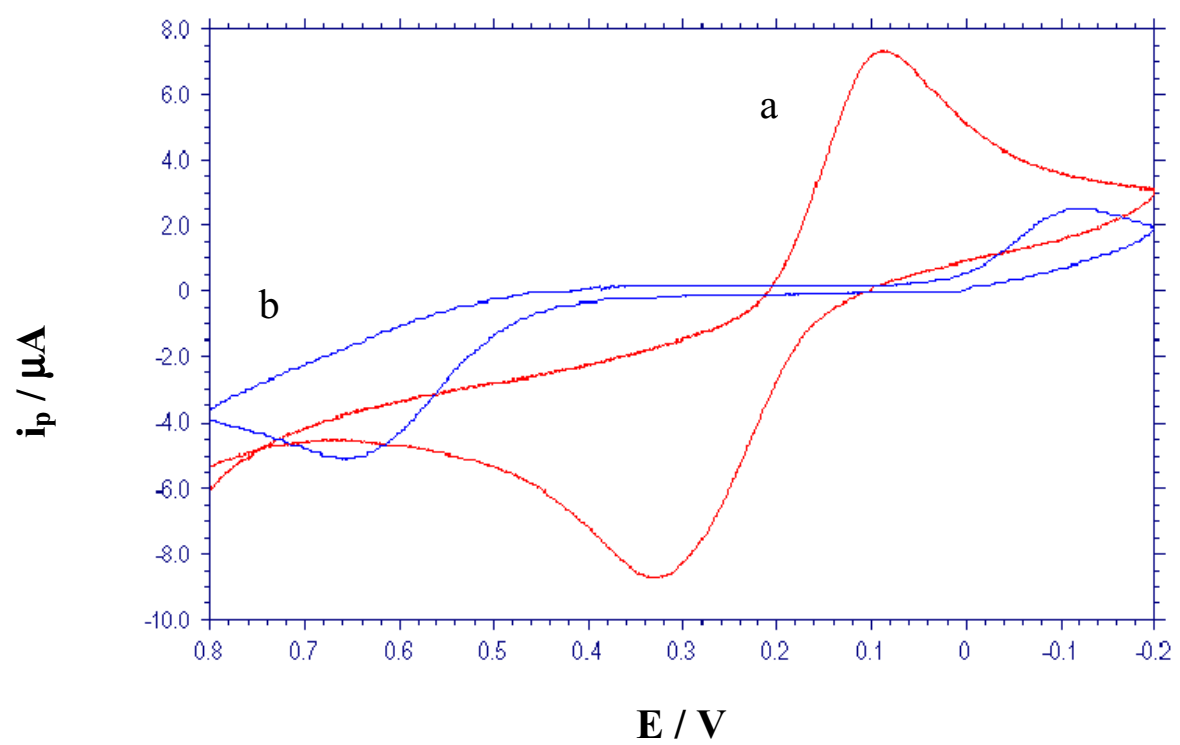

Figure 4. Cyclic voltammograms of hydroquinone on L-Cys SAMs/Au (a) and bare Au (b) electrodes.

Optimization of L-Cys/Au SAMs for the determination of hydroquinone

Optimum response of self-assembled electrode relies on self-assemble time and $\mathrm{pH}$ value of the buffer solutions.

Fig.5 is the plot of catalytic current to hydroquinone against the immersion time of gold electrode in 0.05 M L-cysteine when forming L-Cys/Au SAMs. The maximum current was observed at $\sim 40 \mathrm{~min}$, which means saturation of L-cysteine is reached on gold surface. Considering the reconstruction of the film, an immersion time of $24 \mathrm{~h}$ was chosen for a fresh gold electrode. When immersion time was short, gold surface has not been saturated by L-cysteine, resulting in smaller current.

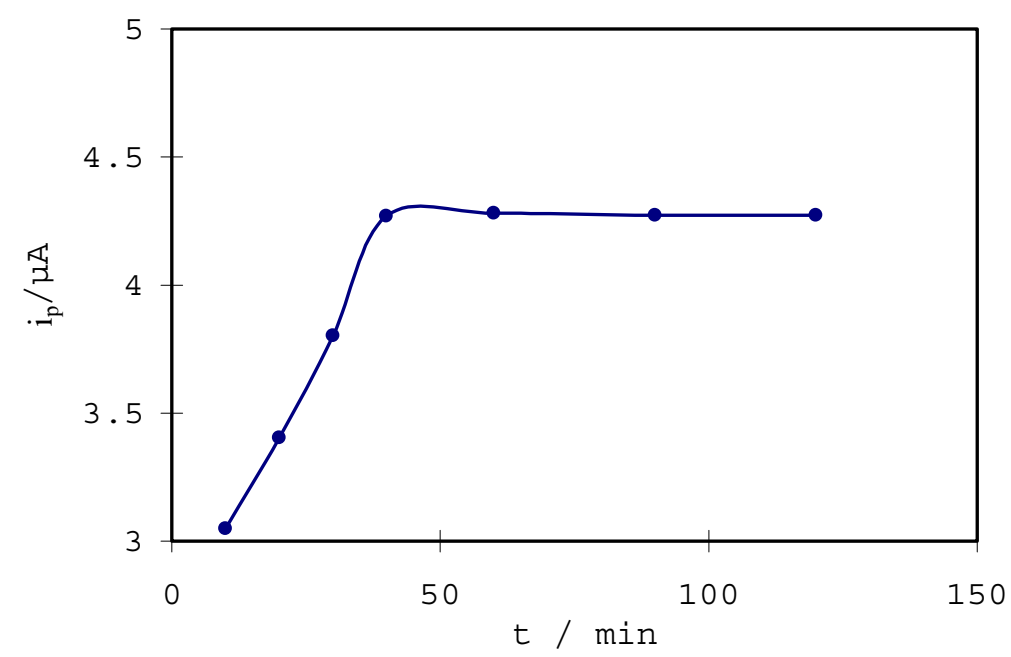

Figure 5. Currents of $1.25 \times 10^{-4} \mathrm{M}$ hydroquinone on L-Cys/Au SAMs at different immersion time. 
The $\mathrm{pH}$ of buffer solution affects protonation of the amino acid and the sensitivity of the electrode. $\mathrm{pH} 4.8$ HAc-NaAc buffer solution was a suitable supporting electrolyte. In the $\mathrm{pH}$ range of 3.5 7, the catalytic current increased until $\mathrm{pH}$ value up to 4.8, while it decreased gradually after that (Fig.6).

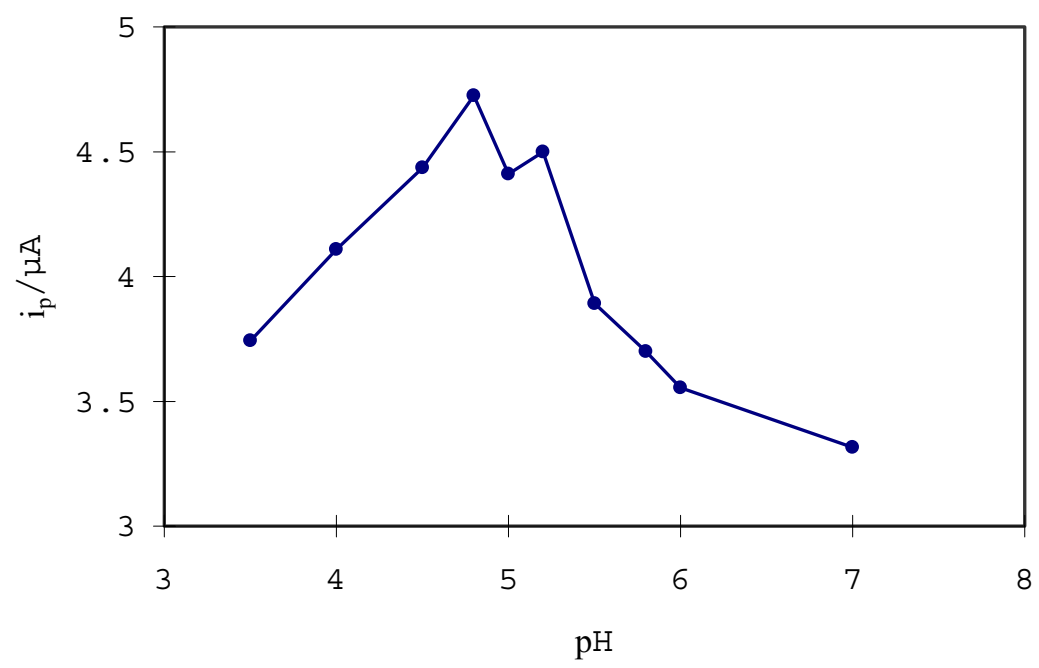

Figure 6. Currents of $1.50 \times 10^{-4} \mathrm{M}$ hydroquinone at L-Cys/Au SAMs at different $\mathrm{pHs}$.

\section{Mechanism for the electrocatalytical process}

The catalytic currents of hydroquinone on L-Cys/Au SAMs changed with the $\mathrm{pH}$ values because $\mathrm{H}^{+}$ participated in the reaction of hydroquinone on electrode, so it is believed that the solution must maintain enough acidity. What's more, L-Cys serving as mediator of the reaction on the electrode was also an electron-transferring accelerator. The mechanism for its acceleration may be explained by hydrogen bonding. The interaction between nitrogen atom of L-Cys and hydrogen atom of phenol hydroxyl in hydroquinone make the bond energy of $\mathrm{O}-\mathrm{H}$ weaken and the hydroxyl activating. Thus, electrons are transferred through N...H-O bond. While at low $\mathrm{pH}, \mathrm{H}^{+}$concentration is so high that $\mathrm{NH}_{2}$ of L-Cys and $\mathrm{O}-\mathrm{H}$ of hydroquinone are existed in the form of $-\mathrm{NH}_{3}{ }^{+}$and $-\mathrm{OH}_{2}{ }^{+}$respectively, which repel one another. Considering largest current and high sensitivity, $\mathrm{pH} 4.8$ is selected.

\section{Calibration and quantitative determination to hydroquinone}

Following the optimization of all conditions, hydroquinone has a good voltammetric response at L$\mathrm{Cys} / \mathrm{Au}$ SAMs. The catalytic current increases linearly with the concentration of hydroquinone in the range of $2.0 \times 10^{-6} \sim 2.0 \times 10^{-4} \mathrm{M}$ (Fig.7). Regression equation is $\mathrm{i}_{\mathrm{pa}}=0.034 \mathrm{c}+0.408\left(\mathrm{i}_{\mathrm{pa}}: \mu \mathrm{A}, \mathrm{c}: \mu \mathrm{M}\right)$, with the correlation coefficient of 0.9986 and the detection limit of $4.0 \times 10^{-7} \mathrm{M}$ by DPV. The RSD of $5.0 \times 10^{-5}$ M hydroquinone signals is $1.6 \%$.

In this analysis, the SAMs electrode was applied to the determination of simulated hydroquinone samples. The results are summarized in Table 1. 


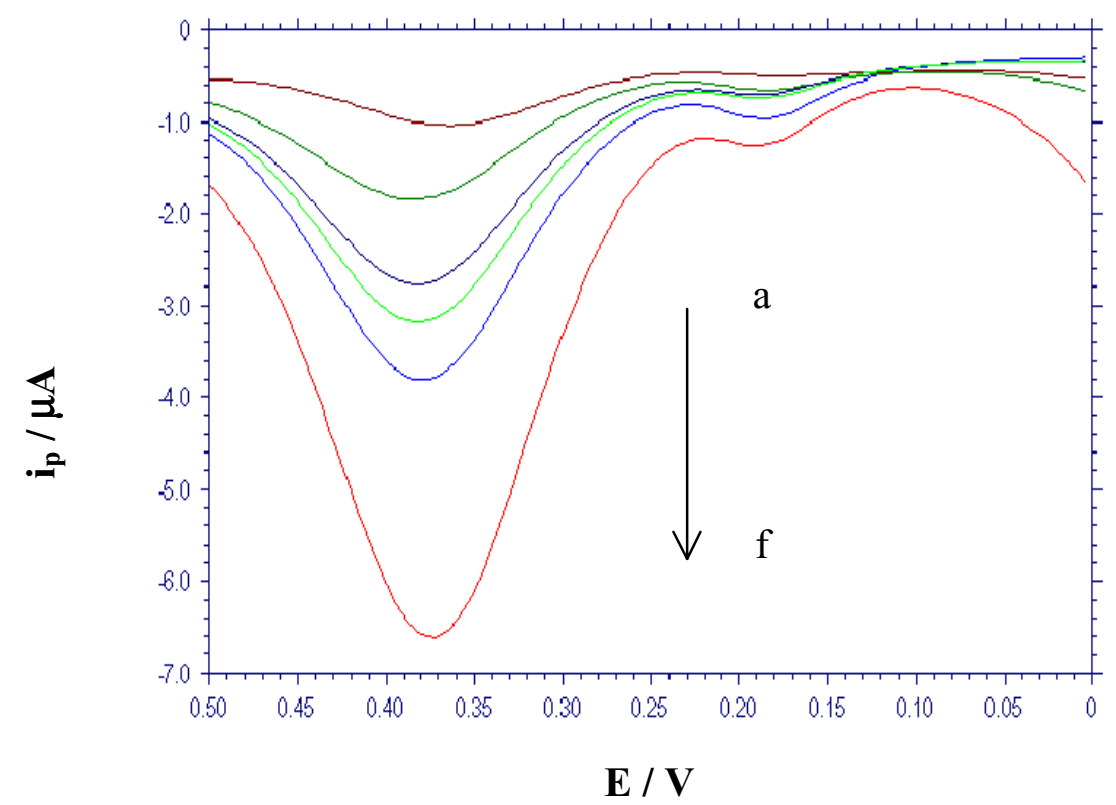

Figure 7. Differential pulse voltammetry of p-benzenediol on L-Cys/Au SAMs.

Table 1. Determination of hydroquinone in simulated samples.

\begin{tabular}{|c|c|c|c|}
\hline Sample(M) & Added(M) & Founded(M) & Recovery(\%) \\
\hline $1.00 \times 10^{-5}$ & $3.00 \times 10^{-5}$ & $3.99 \times 10^{-5}$ & 99.67 \\
\hline $1.00 \times 10^{-5}$ & $3.50 \times 10^{-5}$ & $4.47 \times 10^{-5}$ & 99.14 \\
\hline $1.00 \times 10^{-5}$ & $6.00 \times 10^{-5}$ & $6.87 \times 10^{-5}$ & 97.83 \\
\hline $1.00 \times 10^{-5}$ & $9.00 \times 10^{-5}$ & $1.01 \times 10^{-4}$ & 101.11 \\
\hline $1.00 \times 10^{-5}$ & $1.00 \times 10^{-4}$ & $1.11 \times 10^{-4}$ & 102.00 \\
\hline
\end{tabular}

\section{References}

1. Ulman, A.; Chem. Rev. 1996, 96, 1533.

2. Daniel, M.; Iva, T. Electroanalysis 1996, 8, 207.

3. Wink, Th.; Zuilen, S.J.; Bult, A.; Bennekom, W.P. Analyst 1997, 122, 43R.

4. Widrig, C.A.; Alves, C.A.; Porter, M.D.; J. Am. Chem. Soc. 1991, 113, 8550.

5. Sun, L.; Crooks, R.M.; J. Electrochem. Soc. 1991, 138, 23.

6. Samant, M.G.; Brown, C.A.; Gordon, J.G. Langmuir 1991, 7, 437.

7. Rubinstein, I.; Steinberg, S.; Tor, Y.; Shanzer, A. J. Sagiv. Nature 1988, 332, 426.

8. Steinberg, S.; Tor, Y.; Sabatani, E.; Rubinstein, I. J. Am. Chem. Soc. 1991, 113, 5176.

9. Steinberg, S.; Rubinstein, I. Langmuir 1992, 8, 1183.

10. Turyan, I.; Mandler, D. Anal. Chem. 1994, 66, 56.

11. Turyan, I.; Mandler, D. Anal.Chem. 1997, 69, 894.

12. Berchmans, S.; Yegnaraman, V.; Sandhyarani, N. J. Electroanal. Chem. 1999, 468, 170.

13. He, X.C.; Li, P.; Mo, J.Y. FENXI CESHI XUE BAO (Chinese) 2000, 19(1), 19.

14. Willner, I.; Riklin, A. Anal. Chem. 1994, 66, 1535. 
15. Creager, S.E.; Olsen, K.G. Anal. Chim. Acta. 1995, 307, 277.

16. Jung, S.K.; Wilson, G.S. Anal.Chem. 1996, 68, 591.

17. Uvdal, K.; Bodo, P.; Liedberg, B. J. Colloid Interface Sci. 1992, 149, 162.

18. Ihs, A.; Liedbeg, B. J. Colloid Interface Sci. 1991, 144, 282.

19. Schlereth, D.D.; Katz, E.; Schmidt, H.L. Electroanalysis 1995, 7, 46.

20. Liu, A.C.; Chen, D.C.; Lin, C.C. Anal. Chem. 1999, 71, 1549.

21. Gu, K.; Zhu, J.J.; Chen, H.Y. Chinese J. Anal. Chem. 1999, 27(10), 1172.

22. Zhang, H.M.; Zhu, Z.W.; Li, N.Q. The progress of Electroanal. Chem. (Chinese). Xi'an: Xi'an Map Publishing House.

23. Wu, X.Q.; Wang, Z.S.; Meng, X.Y.; Zhang, Z.R. Electrochemistry (Chinese) 2000, 6(2), 163.

24. Uvdal, K.; Vikinge, T.P. Langmuir 2001, 17(6), 2008.

25. Sluyters-Rehhach, M.; Sluyters, J.H. Electroanalytical Chemistry. Ed. A.J.Bard, 1970, Vol.4, Marcel Dekker, New York.

26. Amatore, C.; Saveant, J.M. Tessier, D. J. Electroanal. Chem. 1983, 147, 39.

27. Sabatani, E.; Rubinstain, I.; Maoz, I. J. Sagiv, J. Electroanal. Chem. 1987, 219, 365.

Sample Availability: Available from the authors.

(C) 2002 by MDPI (http://www.mdpi.net). Reproduction is permitted for noncommercial purposes. 